\title{
EVENTUAL SELF-SIMILARITY OF SOLUTIONS FOR THE DIFFUSION EQUATION WITH NONLINEAR ABSORPTION AND A POINT SOURCE
}

\author{
PETER V. GORDON* AND CYRILL B. MURATOV ${ }^{\dagger}$
}

\begin{abstract}
This paper is concerned with the transient dynamics described by the solutions of the reaction-diffusion equations in which the reaction term consists of a combination of a superlinear power-law absorption and a time-independent point source. In one space dimension, solutions of these problems with zero initial data are known to approach the stationary solution in an asymptotically self-similar manner. Here we show that this conclusion remains true in two space dimensions, while in three and higher dimensions the same conclusion holds true for all powers of the nonlinearity not exceeding the Serrin critical exponent. The analysis requires dealing with solutions that contain a persistent singularity and involves a variational proof of existence of ultra-singular solutions, a special class of self-similar solutions in the considered problem.
\end{abstract}

Key words. Self-similarity, diffusion-absorption, source-sink models, morphogen gradients.

AMS subject classifications. 35C06, 35K61, 35B40, 35Q92

1. Introduction. Many problems of mathematical biology can be modeled by reaction-diffusion equations containing strongly localized source terms. For example, in embryonic development locally produced molecules called morphogens spread through the developing tissue, producing graded concentration profiles that provide positional information guiding morphogenesis 22. The simplest mechanism of morphogen gradient formation involves diffusion and degradation of morphogen molecules throughout the tissue $19,25,29,32,37$. Typically, the degradation is a nonlinear process due to the presence of regulatory feedbacks 2 . In one of the generic regulatory mechanisms the degradation rate is an increasing function of the morphogen concentration, which in the simplest case can be modeled by a power law [9]. After a suitable non-dimensionalization, this mechanism gives rise to the following canonical reaction-diffusion model $9,18,19,25,35,37,38]$ :

$$
u_{t}=\Delta u-u^{p}+\alpha \delta(x) .
$$

Here, $u=u(x, t) \in \mathbb{R}^{+}$and represents the morphogen concentration, $x \in \mathbb{R}^{d}$ is the spatial coordinate, $t \in \mathbb{R}^{+}$is time, $\delta(x)$ is the spatial Dirac delta-function, and $p>1$ and $\alpha>0$ are the order of the degradation reaction and the source strength, respectively. Note that although our interest in this type of equations arose in the context of morphogen gradients 12 14, 23, this equation is quite general and may also arise, for example, in the studies of intracellular calcium dynamics [30, 31], the dynamics of photo-generated charge carriers in semiconductors 17,33, or the kinetics of a non-conserved order parameter in Ginzburg-Landau models of phase transitions in the presence of heterogeneities 15,20 .

In this paper we are interested in the transient dynamics described by 1.1 with zero initial data, $u(x, 0)=0$ (for the precise definition of the solution, see section 2). Recently, we studied this problem in one space dimension, $d=1$ 12,23. In this case we proved that the solution of this problem is monotone increasing in $t$ for any

\footnotetext{
*Department of Mathematics, University of Akron, Akron, Ohio 44325, USA (pgordon@uakron.edu)

${ }^{\dagger}$ Department of Mathematical Sciences, New Jersey Institute of Technology, University Heights, Newark, NJ 07102, USA (muratov@njit.edu)
} 
$x$ and converges to the unique stationary solution. Moreover, we showed that the ratio of the solution of the initial value problem to the stationary solution, written as a function of time $t$ and the parabolic similarity variable $x / \sqrt{t}$, approaches some limit profile independent of $\alpha$ when $t \rightarrow \infty$. This has to do with the existence of an ultra-singular solution for problem [1.1), namely a function $U: \mathbb{R}^{d} \backslash\{0\} \times \mathbb{R}^{+} \rightarrow \mathbb{R}^{+}$ verifying

$$
\begin{cases}U_{t}=\Delta U-U^{p} & (x, t) \in \mathbb{R}^{d} \backslash\{0\} \times \mathbb{R}^{+}, \\ \lim _{t \rightarrow 0} U(x, t)=0 & x \in \mathbb{R}^{d} \backslash\{0\}, \\ \lim _{|x| \rightarrow 0}|x|^{\frac{2}{p-1}} U(x, t)>0 & t \in \mathbb{R}^{+} .\end{cases}
$$

Furthermore, this function is self-similar in the sense that $|x|^{\frac{2}{p-1}} U(x, t)$ can be written as a function of the similarity variable only. In addition, this function is, equivalently, the limit of the solutions of (1.1) with zero initial data as $\alpha \rightarrow \infty$.

Our choice of terminology is motivated by another class of self-similar solutions, called very singular solutions, which arise in a closely related problem that is given by (1.1) with $\alpha=0$ and the initial data in the form of a constant multiple of a deltafunction 7, 10, 11. These solutions satisfy (1.2), except for the last condition, and are instead smooth for all $t>0$, blow up faster than the heat kernel at the origin as $t \rightarrow 0$ and go to zero uniformly as $t \rightarrow \infty$. Moreover, these solutions are, in a suitable sense, the long time attractors of the solutions of the above problem [16. In contrast, the ultra-singular solutions constructed by us contain a persistent singularity at the origin for all $t>0$, as can be seen from the third condition in (1.2), and approach from below the unique stationary solution as $t \rightarrow \infty$. Let us also note that existence of very singular solutions depends delicately on the power $p$ of the nonlinearity and the space dimension $d$. In fact, non-trivial solutions of the problem considered in 7 exist if and only if $p<p_{c}$, where $p_{c}:=(d+2) / d$ is the Fujita critical exponent [5]. In particular, in one space dimension very singular solutions exist if and only if $p<3$. At the same time, as we showed in [12], ultra-singular solutions exist for all $p>1$ in one space dimension.

Coming back to our problem, it is natural to expect that the solutions of 1.1 ) with zero initial data would exhibit the same type of behavior in dimensions $d>$ 1 , namely, that they approach the unique stationary solution of 1.1 from below as $t \rightarrow \infty$, and do so in an asymptotically self-similar fashion. However, in going to arbitrary dimensions one encounters the difficulty that for $d \geq 2$ non-negative stationary solutions that satisfy (1.1) classically away from the origin exist if and only if $p<p^{*}$ [4, 6, 8, 36] (for a recent overview of results on nonlinear elliptic equations involving measures, see 26]), where

$$
p^{*}:= \begin{cases}\infty & d=2, \\ \frac{d}{d-2} & d \geq 3,\end{cases}
$$

is often referred to as the Serrin critical exponent. Therefore, one can only hope to extend our one-dimensional results to the case $d>1$ when $p<p^{*}$. In fact, the latter condition is also necessary for the existence of distributional solutions of 1.1 with a persistent singularity at the origin [3].

In this paper, we study equation (1.1) with zero initial condition, $d \geq 2$ and $1<p<p^{*}$. Under these assumptions we prove that the one-dimensional picture described above extends to higher dimensions. The main difference with the onedimensional case is that all solutions of 1.1 with zero initial data are unbounded 
near the origin for all times $t>0$. Note that singular solutions of 1.1 (with $\alpha \geq 0$ ) have been considered in the literature in a variety of contexts $3,5,7,16,24$. In particular, Baras and Pierre developed a general existence theory for solutions of (1.1) in which the delta-function in the right-hand side is replaced by a general bounded Radon measure on $\mathbb{R}^{d} \times \mathbb{R}^{+}[3$. They provided a necessary and sufficient condition for existence in terms of parabolic capacity.

Although the framework of $[3$ can be straightforwardly extended to the problem under consideration, in view of the special form of the source term in the righthand side of (1.1) we chose to give a self-contained proof of existence, uniqueness, regularity and some qualitative properties of solutions of (1.1) with zero initial data. These results are presented in Theorem 1. We then proceed to establish existence of a self-similar solution that satisfies $(1.2)$. The proof is an adaptation of our variational proof in [12] for the one-dimensional case (for a related variational proof in a different context, see also [10]). The result is contained in Theorem 2 Finally, our main result is on the asymptotic self-similar behavior of solutions of (1.1) with zero initial data given in Theorem 3 . There we prove that the long-time limit of solutions can be characterized by the self-similar solution constructed in Theorem 2 .

From the point of view of the applications, our results apply in two space dimensions irrespectively of the power $p$ of the nonlinearity. In particular, they indicate that the approach of the morphogen concentration to the steady state in a developing epithelium, which to a first approximation is a two-dimensional layer of cells, is asymptotically self-similar and, therefore, exhibits robustness in the case of a point source in the plane. Such robustness was previously demonstrated for these types of problems in one space dimension [9, 14,23. Our results also apply in the case of three space dimensions and a physically important case of a second-order degradation reaction, $p=2$. At the same time, our results break down in the case $d=3$ and $p=3$, corresponding to, e.g., the Ginzburg-Landau equation for the non-conserved order parameter in the presence of a localized heterogeneity [20].

Our paper is organized as follows. In Sec. 22, we introduce the notation used throughout the paper, present a few auxiliary facts and state the main results. Sections 3,4 and 5 are then devoted to the proofs of each the three theorems in Sec. 2. respectively.

2. Preliminaries and the main results. In this section, we introduce the notations, collect a number of known results that will be useful throughout the paper and state our main theorems.

We begin with a discussion of the stationary solutions for (1.1). By a stationary solution for a given $\alpha>0$, we mean a non-negative function $v_{\alpha} \in L^{p}\left(\mathbb{R}^{d}\right)$ which satisfies

$$
-\Delta v_{\alpha}+v_{\alpha}^{p}=\alpha \delta(x) \quad \text { in } \quad \mathcal{D}^{\prime}\left(\mathbb{R}^{d}\right)
$$

Solutions of 2.1) enjoy the following properties:

i) For each $\alpha>0, d \geq 2$ and $1<p<p^{*}$, problem 2.1 has a unique positive solution that belongs to $C^{\infty}\left(\mathbb{R}^{d} \backslash\{0\}\right)$, is radially-symmetric, and behaves as

$$
v_{\alpha}(x) \simeq \alpha \Phi(x), \quad|x| \ll 1
$$


where

$$
\Phi(x):= \begin{cases}\frac{1}{2 \pi} \ln \frac{1}{|x|}, & d=2, \\ \frac{1}{(d-2)\left|\mathbb{S}^{d-1}\right|} \cdot \frac{1}{|x|^{d-2}}, & d \geq 3,\end{cases}
$$

is the fundamental solution of the Laplace's equation.

ii) $v_{\alpha}(x)$ is an increasing function of $\alpha$ for each $x \neq 0$ fixed and approaches from below the function $v_{\infty}(x)$ as $\alpha \rightarrow \infty$, where

$$
v_{\infty}(x):=\frac{c(p, d)}{|x|^{\frac{2}{p-1}}}, \quad c(p, d)=\left[\frac{2}{p-1}\left(\frac{2 p}{p-1}-d\right)\right]^{\frac{1}{p-1}} .
$$

Furthermore, $v_{\infty}(x)$ is the only classical solution of 2.2 on $\mathbb{R}^{d} \backslash\{0\}$ which grows faster than $\Phi(x)$ as $|x| \rightarrow 0$.

For these and other results related to the solutions of 2.2 , we refer the reader to [4,6, 8, 26, 36 and further references therein.

We next turn to assigning the meaning to the solutions of 1.1 with zero initial data. As these solutions are expected to exhibit a singularity of the type $\alpha \Phi(x)$ near the origin, they should be understood in an appropriate distributional sense. To illustrate this point, let us first consider the linearized version of (1.1). Extending the solution by zero for $t \leq 0$ and setting $\alpha=1$ for simplicity, we are lead to the following equation:

$$
I_{t}=\Delta I+\delta(x) \theta(t) \quad \text { in } \quad \mathcal{D}^{\prime}\left(\mathbb{R}^{d+1}\right),
$$

where $\theta(t)$ is the Heaviside step function. The solution of this equation reads

$$
I(x, t)=\int_{0}^{t} \frac{\theta(t)}{[4 \pi(t-s)]^{d / 2}} e^{-\frac{|x|^{2}}{4(t-s)}} d s=\frac{1}{4 \pi^{d / 2}} \frac{\theta(t)}{|x|^{d-2}} \Gamma\left(\frac{d}{2}-1, \frac{|x|^{2}}{4 t}\right),
$$

where $\Gamma(a, x)$ is the incomplete Gamma-function [1]. Note that $I(x, t)$ is a monotonically increasing function of $t$ that approaches $\Phi(x)$ from below for each $x \neq 0$ as $t \rightarrow \infty$ when $d \geq 3$, while it blows up logarithmically for all $x \neq 0$ as $t \rightarrow \infty$ in the case $d=2$. Also note that for $t>0$ we have the following asymptotic behavior of $I(x, t)$ :

$$
I(x, t) \simeq \begin{cases}t^{1-\frac{d}{2}} \Phi(x / \sqrt{t}), & |x| \ll \sqrt{t}, \\ 2^{2-d} \pi^{-d / 2} t^{2-\frac{d}{2}} e^{-\frac{|x|^{2}}{4 t}}|x|^{-2}, & |x| \gg \sqrt{t} .\end{cases}
$$

In particular, a straightforward calculation shows that $I(\cdot, t) \in L^{p}\left(\mathbb{R}^{d}\right)$ for every $t>0$, provided that $1 \leq p<p^{*}$. This dictates that for those values of $p$ the nonlinear term in (1.1) is expected to be "dominated" by the delta-function near the origin. The latter observation is key to the well-posedness of the initial value problem for (1.1).

We next introduce the definition of solutions of (1.1) with zero initial data as distributions for which the nonlinearity in (1.1) also makes sense.

DeFINITION 2.1. We call $u(x, t)$ a solution of (1.1) with zero initial data, if for any $T>0$ the map $t \mapsto u(\cdot, t)$ belongs to $C\left([0, T] ; L^{p}\left(\mathbb{R}^{d}\right)\right), u(\cdot, 0)=0$ and

$$
\int_{0}^{T} \int_{\mathbb{R}^{d}} u\left(\varphi_{t}+\Delta \varphi-|u|^{p-1} \varphi\right) d x d t+\alpha \int_{0}^{T} \varphi(0, t) d t=0 \quad \forall \varphi \in C_{c}^{\infty}\left(\mathbb{R}^{d} \times(0, T)\right) .
$$


Our first result concerns existence, uniqueness, regularity and qualitative properties of positive solutions from Definition 2.1 (see also the general framework in [3]).

THEOREM 1. For each $\alpha>0, d \geq 2$ and $1<p<p^{*}$, there is a unique positive solution $u(x, t)$ of (1.1) with zero initial data in the sense of Definition 2.1. Moreover, it is radially-symmetric, solves

$$
u_{t}=\Delta u-u^{p}
$$

classically for all $(x, t) \in \mathbb{R}^{d} \backslash\{0\} \times \mathbb{R}^{+}$and obeys $u(x, t) \simeq \alpha \Phi(x)$ for all $t>0$ and $|x| \ll 1$. In addition, for each $x \neq 0$ the map $t \mapsto u(x, t)$ is non-decreasing, and $u(x, t) \rightarrow v_{\alpha}(x)$ from below as $t \rightarrow \infty$.

Our next result concerns self-similar solutions of $[1.2)$ that are constructed via the similarity ansatz

$$
U(x, t)=v_{\infty}(x) \phi(\zeta), \quad \zeta=\ln \left(\frac{|x|}{\sqrt{t}}\right) .
$$

Here $0 \leq \phi(\zeta) \leq 1$ is some unknown function, which will be referred to as the selfsimilar profile. Substituting the similarity ansatz from (2.10) into (1.2), after some algebra we obtain the following equation for the self-similar profile $\phi$ :

$$
\phi^{\prime \prime}+\left(\frac{e^{2 \zeta}}{2}-\frac{p+3}{p-1}+d-1\right) \phi^{\prime}+\frac{2}{p-1}\left(\frac{p+1}{p-1}-d+1\right)\left(\phi-\phi^{p}\right)=0, \quad \zeta \in \mathbb{R},
$$

supplemented with the limit behavior

$$
\begin{array}{ll}
\lim _{\zeta \rightarrow-\infty} \phi(\zeta)=1, & \lim _{\zeta \rightarrow-\infty} \frac{d \phi(\zeta)}{d \zeta}=0, \\
\lim _{\zeta \rightarrow+\infty} \phi(\zeta)=0, & \lim _{\zeta \rightarrow+\infty} \frac{d \phi(\zeta)}{d \zeta}=0 .
\end{array}
$$

Solutions of (2.11) satisfying 2.12 and $(2.13)$ will be sought as weak solutions belonging, after subtracting a function $\eta \in C^{\infty}(\mathbb{R})$ that obeys

$$
\eta^{\prime}(\zeta) \leq 0, \quad \zeta \in \mathbb{R}, \quad \eta(\zeta)=1, \quad \zeta \in(-\infty, 0], \quad \eta(\zeta)=0, \quad \zeta \in[1,+\infty),
$$

to the weighted Sobolev space $H^{1}(\mathbb{R}, d \mu)$, which is defined as the completion of the family of smooth functions with compact support with respect to the Sobolev norm

$$
\|w\|_{H^{1}(\mathbb{R}, d \mu)}^{2}:=\left\|w_{\zeta}\right\|_{L^{2}(\mathbb{R}, d \mu)}^{2}+\|w\|_{L^{2}(\mathbb{R}, d \mu)}^{2},
$$

where $\|w\|_{L^{2}(\mathbb{R}, d \mu)}^{2}:=\int_{\mathbb{R}} w^{2}(\zeta) d \mu(\zeta)$, and the measure $\mu$ is defined as

$$
d \mu(\zeta):=\rho(\zeta) d \zeta, \quad \rho(\zeta):=\exp \left\{\frac{e^{2 \zeta}}{4}-\left(\frac{p+3}{p-1}-d+1\right) \zeta\right\}
$$

This setting allows to view 2.11) as the Euler-Lagrange equation of a certain energy functional and prove existence of solutions via the direct method of calculus of variations (compare also with 10$]$ ). We have the following result.

THEOREM 2. For each $d \geq 2$ and $1<p<p^{*}$, there exists a unique weak solution $\phi \in H_{\text {loc }}^{1}(\mathbb{R})$ of 2.11 such that $\phi-\eta \in H^{1}(\mathbb{R}, d \mu)$ and $0 \leq \phi \leq 1$. Furthermore, 
$\phi \in C^{\infty}(\mathbb{R})$, satisfies (2.11) classically, and $0<\phi<1$. In addition, $\phi$ is strictly decreasing, satisfies 2.12) and 2.13), and has the following asymptotic behavior:

$$
\phi(\zeta) \sim \exp \left\{-\frac{e^{2 \zeta}}{4}+\left(\frac{5-p}{p-1}-d+1\right) \zeta\right\}, \quad \zeta \rightarrow+\infty .
$$

Finally, the main result of this paper is the following.

THEOREM 3. For $\alpha>0, d \geq 2$ and $1<p<p^{*}$, let $u(x, t)$ be the solution of 1.1p with zero initial data, and define

$$
F(\zeta, t):=\frac{u(x, t)}{v_{\alpha}(x)}, \quad \zeta=\ln \left(\frac{|x|}{\sqrt{t}}\right) .
$$

Then

$$
F(\zeta, t) \rightarrow \phi(\zeta) \text { as } t \rightarrow \infty
$$

where $\phi(\zeta)$ is as in Theorem 2

3. Proof of Theorem 1. In this section, we prove existence of distributional solutions of 1.1 with zero initial data, as well as their uniqueness, regularity, asymptotic behavior at the origin and monotonic approach from below to the solution of 2.1. As we already noted in the introduction, existence of these singular solutions can be treated within the general framework developed in [3]. For the sake of completeness, we give a self-contained proof that uses the special form of the measure appearing in (1.1) and follows the ideas used in the analysis of the elliptic case (for an overview, see, e.g., 26 ).

To prove existence of solutions of (1.1) with zero initial data in the sense of Definition 2.1, we mollify the delta-function and consider for each $n \in \mathbb{N}$ the solution $u=u_{n}(x, t)$ that vanishes at $t=0$ of the equation

$$
u_{t}=\Delta u-f_{n}(u)+\alpha g_{n}(x), \quad(x, t) \in \mathbb{R}^{d} \times \mathbb{R}^{+},
$$

where $g_{n}(x)=n^{d} g(n x)$ for some non-negative, radially-symmetric function $g \in$ $C_{c}^{\infty}\left(\mathbb{R}^{d}\right)$ supported on a unit ball and satisfying $\int_{\mathbb{R}^{d}} g(x) d x=1$. Also, here $f_{n} \in$ $\operatorname{Lip}(\mathbb{R})$ is the extended and truncated nonlinearity, namely,

$$
f_{n}(u):= \begin{cases}0, & u<0 \\ u^{p}, & 0 \leq u \leq \bar{u}_{n}, \quad \bar{u}_{n}:=\left(\alpha\left\|g_{n}\right\|_{L^{\infty}\left(\mathbb{R}^{d}\right)}\right)^{1 / p} . \\ \bar{u}_{n}^{p}, & u>\bar{u}_{n},\end{cases}
$$

Short-time existence of a unique solution $u_{n}$ of (3.1) in the class of bounded uniformly continuous functions then follows from the classical theory (see, e.g., [34, Chapter 15], [21]). Moreover, since $u=0$ and $u=\bar{u}_{n}$ are a sub- and a super-solution for (3.1), the solution is, in fact, global in time, and we have $f_{n}\left(u_{n}\right)=u_{n}^{p}$. By standard parabolic theory, this solution is smooth for all $t \geq 0$. Also, by construction the solution is radially-symmetric. In addition, by comparison principle [27, for all $t>0$ the solution is positive and monotonically increasing in $t$ for each $x \in \mathbb{R}^{d}$. In particular, we have $u_{n}(x, t) \rightarrow v_{\alpha, n}(x)>0$ from below for all $x \neq 0$ as $t \rightarrow \infty$, where $v_{\alpha, n}$ solves

$$
-\Delta v_{\alpha, n}+v_{\alpha, n}^{p}=\alpha g_{n}(x) .
$$


Furthermore, it is easy to see that $v_{\alpha, n} \leq \bar{v}_{\alpha, n}$, where $\bar{v}_{\alpha, n}(x):=C|x|^{-\frac{2}{p-1}}$, for some $C>0$ depending only on $d, p, \alpha$ and $\|g\|_{L^{\infty}\left(\mathbb{R}^{d}\right)}$. Indeed, choosing $C$ sufficiently large, we have that the left-hand side of $(3.3)$ is bounded from below by $\frac{1}{2} C^{p}|x|^{-\frac{2 p}{p-1}} \geq$ $\alpha\|g\|_{L^{\infty}\left(\mathbb{R}^{d}\right)}|x|^{-d} \geq \alpha g_{n}(x)$ for all $|x| \leq \frac{1}{n}$, as long as $p<p^{*}$. At the same time, trivially $\frac{1}{2} C^{p}|x|^{-\frac{2 p}{p-1}} \geq 0=\alpha g_{n}(x)$ for all $|x|>\frac{1}{n}$. Hence $\bar{v}_{\alpha, n}$ is a super-solution, and the conclusion follows by maximum principle 28 .

We now show that

$$
u_{n}(x, t) \leq \alpha C_{d} I(x, 1+2 t) \quad \forall(x, t) \in \mathbb{R}^{d} \times \mathbb{R}^{+},
$$

for some $C_{d}>0$ depending only on the dimension and the choice of $g$, where $I(x, t)$ is defined in (2.6). Indeed, by comparison principle $u_{n}$ can be estimated from above by the solution of the linearized equation:

$$
u_{n}(x, t) \leq \alpha \int_{\mathbb{R}^{d}} I(x-y, t) g_{n}(y) d y \quad \forall(x, t) \in \mathbb{R}^{d} \times \mathbb{R}^{+} .
$$

Therefore, recalling the definitions of $g_{n}$ and $I$, we have

$$
\begin{aligned}
u_{n}(x, t) & \leq \alpha I(x, 1+2 t) \int_{\mathbb{R}^{d}} \frac{I(x-y, t)}{I(x, 1+2 t)} g_{n}(y) d y \\
& \leq \alpha I(x, 1+2 t)\|g\|_{L^{\infty}\left(\mathbb{R}^{d}\right)} \int_{B_{1}(0)} \frac{I\left(\tilde{x}-\tilde{y}, n^{2} t\right)}{I\left(\tilde{x}, n^{2}(1+2 t)\right)} d \tilde{y},
\end{aligned}
$$

where $\tilde{x}:=n x$. It is then not difficult to see from (2.7) that the integral in the right-hand side of (3.6) is bounded independently of $\tilde{x}, t$ and $n$, which yields the claim.

The estimate in (3.4) and the fact that $p<p^{*}$ imply that $u_{n}(\cdot, t)$ are uniformly bounded in $L^{p}\left(\mathbb{R}^{d}\right)$ for all $t \in[0, T]$, with $T>0$ arbitrary. Therefore, the right-hand side of (3.1) is uniformly bounded in $L^{1}\left(\mathbb{R}^{d}\right)$ for each $t \in[0, T]$, and by parabolic theory [34, Chapter 15] we also have that $u_{n}(\cdot, t)$ is uniformly bounded in $W^{1, q}\left(\mathbb{R}^{d}\right)$ for any $1 \leq q<d /(d-1)$. Choosing $q$ sufficiently close to $d /(d-1)$, which is, again, possible for $p<p^{*}$, and passing to a subsequence (not relabeled), by RellichKondrachov theorem we then conclude that $u_{n}(\cdot, t) \rightarrow u(\cdot, t)$ strongly in $L_{l o c}^{p}\left(\mathbb{R}^{d}\right)$ as $n \rightarrow \infty$ for every $t \in[0, T]$. In fact, the bound in $(3.4)$ implies that convergence is in $L^{p}\left(\mathbb{R}^{d}\right)$. In addition, by Sobolev embedding and parabolic theory we have uniform Hölder continuity of $u_{n}(\cdot, t)$ in $L^{p}\left(\mathbb{R}^{d}\right)$ 21. Chapter 4] and, hence, upon extraction of a subsequence we have $u_{n} \rightarrow u$ in $C\left([0, T] ; L^{p}\left(\mathbb{R}^{d}\right)\right)$. Thus, we can pass to the limit in the distributional formulation of (3.1) to obtain that $u$ satisfies (2.8). By construction, $u(\cdot, t)$ is radial. By the same line of arguments, we also have $v_{\alpha, n} \rightarrow v_{\alpha}$ strongly in $L^{p}\left(\mathbb{R}^{d}\right)$ as $n \rightarrow \infty$.

Uniqueness of solutions of 2.8 follows from parabolic theory, noting that the difference $w(x, t)$ of any two solutions of 2.8 satisfies

$$
w_{t}=\Delta w+k(x, t) w \quad \text { in } \quad \mathcal{D}^{\prime}\left(\mathbb{R}^{d} \times \mathbb{R}^{+}\right),
$$

where $k(\cdot, t)$ is uniformly bounded in $L^{p^{\prime}}\left(\mathbb{R}^{d}\right)$, with $p^{\prime}=p /(p-1)$, for all $t \in[0, T]$, and is, hence, zero. Note that uniqueness also implies that $u$ is the full limit of $u_{n}$ as $n \rightarrow \infty$. Regularity outside the origin is also standard in view of the estimate in (3.4), which implies that $u(\cdot, t)$ is uniformly bounded on $\mathbb{R}^{d} \backslash B_{R}(0)$ for any $R>0$. 
Furthermore, by the bootstrap argument $u_{n}(\cdot, t)$ converges to $u(\cdot, t)$ uniformly in $\mathbb{R}^{d} \backslash B_{R}(0)$ as $n \rightarrow \infty$. In particular, the limit $u(x, t)$ of $u_{n}(x, t)$ is non-decreasing in $t$ for all $x \neq 0$ and approaches some stationary solution $v(x) \leq \limsup _{n \rightarrow \infty} v_{\alpha, n}(x)$ as $t \rightarrow \infty$. At the same time, by elliptic regularity we also have $v_{\alpha, n}(x) \rightarrow v_{\alpha}(x)$ for all $x \in \mathbb{R}^{d} \backslash\{0\}$ as $n \rightarrow \infty$. It then follows that $v(x) \leq v_{\alpha}(x)$.

Finally, the asymptotic behavior of $u(\cdot, t)$ near the origin follows from (2.7) and the fact that $u-\alpha I$ solves forced heat equation with the forcing term of order $|x|^{-s}$ near the origin for some $0<s<d$ and is, therefore, less singular than $\Phi$ there. Combining this with monotonicity of $u(x, t)$ in $t$ and 2.7 , we then conclude that $u(x, t) \simeq \alpha \Phi(x)$ for $|x| \ll 1$ uniformly in $t$, which implies that $v=v_{\alpha}$.

4. Proof of Theorem 2, Observe that 2.11 is the Euler-Lagrange equation for the energy functional

$$
\begin{aligned}
\mathcal{E}[\phi]:= & \int_{\mathbb{R}}\left\{\frac{1}{2}\left(\frac{d \phi}{d \zeta}\right)^{2}+\left(\frac{1}{p-1}-\frac{d-1}{p+1}\right) \eta\right. \\
& \left.-\left(\frac{1}{(p-1)^{2}}-\frac{d-1}{p^{2}-1}\right) \phi^{2}\left(p+1-2 \phi^{p-1}\right)\right\} d \mu(\zeta),
\end{aligned}
$$

which is well-defined for all $\phi-\eta \in H^{1}(\mathbb{R}, d \mu)$. This energy can be written as

$$
\mathcal{E}[\phi]=\int_{\mathbb{R}}\left\{\frac{1}{2}\left(\frac{d \phi}{d \zeta}\right)^{2}+\left(\frac{p+1}{p-1}-K(d)\right)\left[\frac{\eta}{p+1}-\frac{\phi^{2}\left(p+1-2 \phi^{p-1}\right)}{p^{2}-1}\right]\right\} d \mu(\zeta),
$$

where

$$
K(d)= \begin{cases}1 & d=2 \\ \frac{p^{*}+1}{p^{*}-1} & d \geq 3\end{cases}
$$

Clearly, the term multiplying the square bracket in 4.2 is positive for all $p<p^{*}$. This means that we can apply the proof in [12, Theorem 2.1] verbatim to prove existence and qualitative properties of solutions of (2.11) in the considered class of functions. In particular, the decay estimate follows as in [12, Remark 1]. $\square$

5. Proof of Theorem 3. We start with the following simple observation. Letting $R>0$, consider the problem

$$
\begin{cases}w_{t}=w_{r r}+\frac{d-1}{r} w_{r}-w^{p} & (r, t) \in(R, \infty) \times(0, \infty) \\ w(R, t)=u(R, t) & t \in(0, \infty) \\ w(r, 0)=0 & r \in(R, \infty)\end{cases}
$$

where, with some abuse of notation, for any $r>0$ we take $u(r, t)$ to be the value of the solution $u(x, t)$ of $(1.1)$ with $|x|=r$. By regularity of $u(R, t)$ (see Theorem 1 ) and standard parabolic theory, problem (5.1) has a unique classical solution. Therefore, by uniqueness we have $w(r, t)=u(r, t)$ for $r \geq R$ and $t \geq 0$. The advantage of considering problem (5.1) instead of 1.1 is that solutions of (5.1) are classical and thus classical comparison principle applies. We also note that the stationary version of (5.1):

$$
\left\{\begin{array}{l}
-W_{r r}-\frac{d-1}{r} W_{r}+W^{p}=0 \quad r \in(R, \infty) \\
W(R)=v_{\alpha}(R)
\end{array}\right.
$$


where, with the same abuse of notation, $v_{\alpha}(r)$ is the solution of 2.1) written as a function of the radial coordinate, has a unique solution satisfying $W(r) \rightarrow 0$ as $r \rightarrow \infty$ (see, e.g., 28, Theorem 4.3.2]). Therefore, $W(r)=v_{\alpha}(r)$ for $r \geq R$.

The proof of Theorem 3 is based on a construction of explicit sub- and supersolutions for problem (5.1). Let

$$
N[w]:=w_{t}-w_{r r}-\frac{d-1}{r} w_{r}+w^{p} .
$$

We say that $\bar{w}$ is a super-solution of $w$ if it solves the differential inequality

$$
\begin{cases}N[\bar{w}] \geq 0 & (r, t) \in(R, \infty) \times(0, \infty) \\ \bar{w}(R, t) \geq u(R, t) & t \in(0, \infty) \\ \bar{w}(r, 0) \geq 0 & r \in(R, \infty)\end{cases}
$$

Similarly, we say that $\underline{w}$ is a sub-solution of $w$ if

$$
\begin{cases}N[\underline{w}] \leq 0 & (r, t) \in(R, \infty) \times(0, \infty) \\ \underline{w}(R, t) \leq u(R, t) & t \in(0, \infty) \\ \underline{w}(r, 0) \leq 0 & r \in(R, \infty)\end{cases}
$$

Then by classical comparison principle 27,28 the solution of problem (5.1) obeys

$$
\underline{w}(r, t) \leq w(r, t) \leq \bar{w}(r, t) \quad(r, t) \in[R, \infty) \times[0, \infty) .
$$

Likewise, we define radial sub- and super-solutions for (5.2). Namely, let

$$
M[W]=-W_{r r}-\frac{d-1}{r} W_{r}+W^{p}
$$

Then the functions $\bar{W}$ and $\underline{W}$ are called super- and sub-solutions of $(5.2)$, respectively, if they satisfy

$$
\left\{\begin{array}{l}
M[\bar{W}] \geq 0 \quad r \in(R, \infty) \\
\bar{W}(R) \geq v_{\alpha}(R)
\end{array}\right.
$$

and

$$
\left\{\begin{array}{l}
M[\underline{W}] \leq 0 \\
\underline{W}(R) \leq v_{\alpha}(R)
\end{array} \quad r \in(R, \infty)\right.
$$

respectively. Again, by the maximum principle 28] we have

$$
\underline{W}(r) \leq W(r) \leq \bar{W}(r) \quad r \in[R, \infty) .
$$

In the following lemma, we construct explicitly a sub- and a super-solution for the stationary problem 5.2 .

Lemma 5.1. Let $R>0$ and $\alpha>0$. Then there exists $0<\bar{\gamma}<1$ such that for all $\bar{\gamma} \leq \gamma<1$ there exists $b>0$ such that the function

$$
v_{0}(r):=\frac{c(p, d)}{\left(r+b r^{\gamma}\right)^{\frac{2}{p-1}}}
$$

is a sub-solution for problem (5.2). Moreover, the function $v_{\infty}(r)$ defined by (2.4) is a super-solution for this problem. 
Proof. Let us start with the construction of a sub-solution. First, observe that direct substitution of $v_{0}$ defined by (5.11) into (5.7) gives

$$
\begin{aligned}
& M\left[v_{0}\right]=\frac{2 b c(p, d)}{(p-1)^{2}\left(r+b r^{\gamma}\right)^{\frac{2 p}{p-1}}}\left\{\gamma b[(d-2)(p-1)-2 \gamma] r^{2(\gamma-1)}\right. \\
& \left.+(p-1)\left[\gamma^{2}-\left(\frac{4 p}{p-1}-d\right) \gamma+d-1\right] r^{\gamma-1}\right\}
\end{aligned}
$$

In order for $v_{0}$ to satisfy the condition $M\left[v_{0}\right] \leq 0$, it is sufficient to choose the parameter $\gamma$ such that the following two inequalities hold:

$$
\gamma \geq \gamma_{1}:=\frac{(d-2)(p-1)}{2}
$$

and

$$
s(\gamma) \leq 0,
$$

where

$$
s(\gamma)=\gamma^{2}-\left(\frac{4 p}{p-1}-d\right) \gamma+d-1
$$

Indeed, first observe that $\gamma_{1}=0$ for $d=2$ and $\gamma_{1}=1-(d-2)\left(p^{*}-p\right) / 2<1$ for $d \geq 3$. Next, since $s(\gamma)$ is a parabola having the following properties: $s(0)=d-1>0$, $s(1)=-\frac{2 d}{p-1}\left(1-\frac{p}{p^{*}}\right)<0$ and $s^{\prime}(\gamma) \leq s^{\prime}(1)<-2 /(p-1)<0$, we conclude that $s(\gamma) \leq 0$ for all $1>\gamma \geq \gamma_{2}$, where $\gamma_{2}<1$ is the smallest root of the equation $s(\gamma)=0$. Setting $\bar{\gamma}=\max \left\{\gamma_{1}, \gamma_{2}\right\}$ we have $M\left[v_{0}\right] \leq 0$. Next, we choose $b$ sufficiently large so that an inequality $v(R) \geq v_{0}(R)$ holds. Thus, both conditions in 5.9 are satisfied, and $v_{0}(r)$ is a sub-solution for problem (5.2).

Now, let us verify that $v_{\infty}(r)$ is a super-solution for $W$. First, by direct substitution we have $M\left[v_{\infty}\right]=0$. Moreover, $v_{\infty}(r)>v_{\alpha}(r)$ for any $\alpha$ and $r>0$ (see section $2)$. Thus, $v_{\infty}(r)$ is a super-solution. $\square$

REMARK 1. It follows from Lemma 5.1 that for each pair $R>0, \alpha>0$ one can choose $0<\gamma<1$ and $b>0$ so that $v_{0}(r)<v_{\alpha}(r)<v_{\infty}(r)$ for all $r \geq R$.

Now we construct a super-solution for problem (5.1).

LEMma 5.2. There exists $T_{1}>0$ such that the function

$$
\bar{w}(r, t):=v_{\infty}(r) \phi(y), \quad y=\log \left(\frac{r}{\sqrt{t+T_{1}}}\right)
$$

is a super-solution of 5.1

Proof. First, observe that $\bar{w}(r, t)=U\left(r, t+T_{1}\right)$ and thus verifies $N[\bar{w}]=0$. Next, since $v_{\infty}(R)>v(R)>u(R, t)$ and $\phi(\zeta) \rightarrow 1$ as $\zeta \rightarrow-\infty$, one can always choose $T_{1}$ large enough, so that $v_{\infty}(R) \phi\left(\log \left(R / \sqrt{t+T_{1}}\right)\right)>u(R, t)$ for all $t>0$. As a result, $\bar{w}$ is a super-solution.

Finally, we give a construction of a sub-solution for problem (5.1). This construction requires the following elementary lemma.

Lemma 5.3. Let $\phi(\zeta)$ be as in Theorem 2. Then, there exists $\zeta_{0}>0$ such that $\phi(\zeta) \leq\left|\phi^{\prime}(\zeta)\right|$ for all $\zeta \geq \zeta_{0}$. 
Proof. First, rewrite 2.11 as follows:

$$
\left[\phi^{\prime}+\left(\frac{e^{2 \zeta}}{2}-\frac{p+3}{p-1}+d-1\right) \phi\right]^{\prime}=\left[e^{2 \zeta}-\frac{2}{p-1}\left(\frac{p+1}{p-1}-d+1\right)\left(1-\phi^{p-1}\right)\right] \phi .
$$

Integrating this equation from $\zeta$ to $\infty$ and taking into account 2.13 and 2.17 , we have

$$
-\phi^{\prime}(\zeta)-\left(\frac{e^{2 \zeta}}{2}-\frac{p+3}{p-1}+d-1\right) \phi(\zeta)>0,
$$

provided that $\zeta$ is sufficiently large. In view of the fact that $\phi^{\prime}(\zeta)<0$ (see Theorem 2), we obtain

$$
\phi(\zeta) \leq 4 e^{-2 \zeta}\left|\phi^{\prime}(\zeta)\right|
$$

for large enough $\zeta$, which gives the desired result. $\square$

Lemma 5.4. Given $R>0$ and $\alpha>0$, there exist $T_{2}>0, b>0$ and $0<\gamma<1$ such that the function

$$
\underline{w}(r, t)= \begin{cases}0 & t<T_{2} \\ v_{0}(r) \phi(z) & t \geq T_{2}\end{cases}
$$

where $v_{0}$ is given by 5.11 and

$$
z=\ln \left(\frac{r+b r^{\gamma}}{\sqrt{t-T_{2}}}\right)
$$

is a sub-solution for problem (5.1).

Proof. Observe first that by 2.17 and the fact that $\phi \in C^{\infty}(\mathbb{R})$ the function defined by (5.20) is smooth for all $r \geq R$ and $t>0$. Next, let us show that $N[\underline{w}] \leq 0$. For $t \leq T_{2}$ this is trivial. For $t>T_{2}$, after a very tedious but straightforward computation, we have:

$$
N[\underline{w}]=-\frac{c(p, d) b}{\left(r+b r^{\gamma}\right)^{\frac{2 p}{p-1}}}\left[\left(-\phi^{\prime}\right)(z) A_{1}(r, z)+\phi(z) A_{2}(r, z)\right],
$$

where

$$
\begin{aligned}
& A_{1}(r, z)=\left(\gamma e^{2 z}-(d-1-\gamma) \delta\right) r^{\gamma-1}+b\left(\frac{\gamma^{2}}{2} e^{2 z}-(d-2) \delta\right) r^{2(\gamma-1)}, \\
& A_{2}(r, z)=\frac{2}{p-1}\left\{\left[2 \gamma\left(\frac{p+1}{p-1}-d+1\right) \phi^{p-1}-(d-1-\gamma) \delta\right] r^{\gamma-1}\right. \\
& \left.+b\left[\gamma^{2}\left(\frac{p+1}{p-1}-d+1\right) \phi^{p-1}-(d-2) \delta\right] r^{2(\gamma-1)}\right\}
\end{aligned}
$$

and

$$
\delta=1-\gamma
$$

Thus, all we need to show is that $\Psi(r, z)=\left(-\phi^{\prime}\right)(z) A_{1}(r, z)+\phi(z) A_{2}(r, z) \geq 0$. In order to show that this condition indeed holds, assume first that $z<\zeta_{0}$, where $\zeta_{0}$ is 
chosen as in Lemma5.3. In this case, by Theorem 2 we have $\phi \geq k_{1}>0$ and $\left|\phi^{\prime}\right| \leq k_{2}$ for some positive constants $k_{1}$ and $k_{2}$. Therefore

$$
\begin{aligned}
& \Psi(r, z) \geq \frac{2}{p-1}\left[2 \gamma\left(\frac{p+1}{p-1}-d+1\right) k_{1}^{p-1}-\left(1+\frac{k_{2}(p-1)}{2}\right)(d-1-\gamma) \delta\right] r^{\gamma-1}+ \\
& \frac{2 b}{p-1}\left[\gamma^{2}\left(\frac{p+1}{p-1}-d+1\right) k_{1}^{p-1}-\left(1+\frac{k_{2}(p-1)}{2}\right)(d-2) \delta\right] r^{2(\gamma-1)}
\end{aligned}
$$

and thus $\Psi(r, z) \geq 0$ independently of $b$ and $T_{2}$ for all $r \geq R$ and $z<\zeta_{0}$, provided that $\delta$ is sufficiently small. In the case when $z \geq \zeta_{0}$ we have, by Lemma 5.3 , that $\phi<\left|\phi^{\prime}\right|$, which implies

$$
\begin{aligned}
& \Psi(r, z) \geq\left|\phi^{\prime}(z)\right|\left[\left(\gamma-\left(1+\frac{2}{p-1}\right)(d-1-\gamma) \delta\right) r^{\gamma-1}\right. \\
& \left.+b\left(\frac{\gamma^{2}}{2}-\left(1+\frac{2}{p-1}\right)(d-2) \delta\right) r^{2(\gamma-1)}\right]
\end{aligned}
$$

and thus $\Psi(r, z) \geq 0$ for all $r \geq R$ and $z \geq \zeta_{0}$ for $\delta$ small enough independently of $b$ and $T_{2}$. Therefore, we conclude that one can choose $\gamma<1$ sufficiently close to unity so that $N[\underline{w}] \leq 0$ for all $r \geq R$ and $t>0$ independently of $b$ and $T_{2}$.

Now we choose $T_{2}$ and $b$ large enough so that $u(R, t)>2 v(R) / 3$ for $t>T_{2}$ and $v_{0}(R)=v(R) / 2$, which is always possible, since by Theorem 1 for each $R>0$ the function $u(R, t)$ is a monotone increasing function of $t$ and $\lim _{t \rightarrow \infty} u(R, t)=v(R)$, and $v_{0}$ is a decreasing function of $b$ that vanishes as $b \rightarrow \infty$. In view of this observation and the fact that $\phi<1$, we have

$$
\underline{w}(R, t)<u(R, t),
$$

for all $t>0$. As a result, $\underline{w}$ given by 5.20 is a sub-solution for 5.1 .

Proof of Theorem 3. Recall first that by construction

$$
\underline{w}(r, t) \leq u(r, t) \leq \bar{w}(r, t),
$$

for all $t \geq 0$ and $r \geq R$. Therefore, by Lemmas 5.4 and 5.2 , for $t>T_{2}$ and $r>R$ we have

$$
\underline{w}(r, t) / v_{\alpha}(r) \leq F(\zeta, t) \leq \bar{w}(r, t) / v_{\alpha}(r),
$$

where $F$ and $\zeta$ and defined by (2.18). Using this inequality, after straightforward algebraic computations we obtain

$$
\begin{aligned}
& F(\zeta, t) \leq \frac{v_{\infty}(r)}{v_{\alpha}(r)} \phi\left(\zeta-\frac{1}{2} \ln \left(1+T_{1} / t\right)\right) \\
& F(\zeta, t) \geq \frac{v_{0}(r)}{v_{\alpha}(r)} \phi\left[\zeta+\ln \left(\frac{1+b e^{-(1-\gamma) \zeta} / t^{(1-\gamma) / 2}}{\sqrt{1-T_{2} / t}}\right)\right] .
\end{aligned}
$$

It is also not difficult to see that

$$
H(\zeta, t):=v_{0}(r) / v_{\infty}(r)=\frac{1}{1+b e^{-(1-\gamma) \zeta / t^{(1-\gamma) / 2}}} .
$$

Taking the limit as $t \rightarrow \infty$ in 5.31, 5.32 and (5.33), we obtain 2.19). 
Acknowledgements. This work was supported, in part, by NSF via grant DMS1119724. CBM was also partially supported by NSF via grants DMS-0908279 and DMS-1313687. The work of PVG was also partially supported by a grant 317882 from the Simons Foundation, and by the US-Israel Binational Science Foundation via grant 2012057. The authors are grateful to V. V. Matveev and V. Moroz for valuable discussions, and to S. Y. Shvartsman for suggesting this problem to us.

\section{REFERENCES}

[1] M. Abramowitz And I. Stegun, eds., Handbook of mathematical functions, National Bureau of Standards, 1964.

[2] H. L. Ashe And J. BRiscoe, The interpretation of morphogen gradients, Development, 133 (2006), pp. 385-394.

[3] P. Baras and M. Pierre, Problèmes paraboliques semi-linéaires avec données mesures, Applicable Anal., 18 (1984), pp. 111-149.

[4] P. BÉnilan And H. Brezis, Nonlinear problems related to the Thomas-Fermi equation, J. Evol. Equ., 3 (2004), pp. 673-770.

[5] H. Brezis And A. Friedman, Nonlinear parabolic equations involving measures as initial conditions, J. Math. Pures Appl., 62 (1983), pp. 73-97.

[6] H. Brezis And L. Oswald, Singular solutions for some semilinear elliptic equations, Arch. Rational Mech. Anal., 99 (1987), pp. 249-259.

[7] H. Brezis, L. A. Peletier, and D. Terman, A very singular solution of the heat equation with absorption, Arch. Rational Mech. Anal., 95 (1986), pp. 185-209.

[8] H. Brezis And L. VÉRon, Removable singularities for some nonlinear elliptic equations, Arch. Rational Mech. Anal., 75 (1980), pp. 1-6.

[9] A. Eldar, D. Rosin, B. Z. Shilo, and N. Barkai, Self-enhanced ligand degradation underlies robustness of morphogen gradients, Devel. Cell, 5 (2003), pp. 635-646.

[10] M. Escobedo AND O. Kavian, Variational problems related to self-similar solutions of the heat equation, Nonlinear Anal., 11 (1987), pp. 1103-1133.

[11] V. A. Galaktionov, S. P. Kurdyumov, and A. A. Samarskil, Asymptotic "eigenfunctions" of the Cauchy problem for a nonlinear parabolic equation, Mat. Sb. (N.S.), 126 (1985), pp. 435-472.

[12] P. V. Gordon And C. B. Muratov, Self-similarity and long-time behavior of solutions of the diffusion equation with nonlinear absorption and a boundary source, Netw. Heterog. Media, 7 (2012), pp. 767-780.

[13] P. V. Gordon, C. B. Muratov, and S. Y. Shvartsman, Local accumulation time in multidimensional sink-source models of morphogen gradients, J. Chem. Phys., 138 (2013), pp. 104121 pp. 1-6.

[14] P. V. Gordon, C. Sample, A. M. Berezhkovskit, C. B. Muratov, and S. Y. Shvartsman, Local kinetics of morphogen gradients, Proc. Natl. Acad. Sci. US., 108 (2011), pp. 61576162.

[15] P. C. Hohenberg And B. I. Halperin, Theory of dynamic critical phenomena, Rev. Mod. Phys., 49 (1977), pp. 435-479.

[16] S. Kamin And L. A. Peletier, Singular solutions of the heat equation with absorption, Proc. Amer. Math. Soc., 95 (1985), pp. 205-210.

[17] B. S. Kerner and V. V. Osipov, Autosolitons, Kluwer, Dordrecht, 1994.

[18] A. Kicheva, P. Pantazis, T. Bollenbach, Y. Kalaidzidis, T. Bittig, F. Julicher, and M. Gonzalez-Gaitan, Kinetics of morphogen gradient formation, Science, 315 (2007), pp. 521-525.

[19] A. D. Lander, W. C. Lo, Q. Nie, and F. Y. WAN, The measure of success: constraints, objectives, and tradeoffs in morphogen-mediated patterning, Cold Spring Harbor Perspectives in Biology, 1 (2009), p. a002022.

[20] A. Levanyuk, V. Osipov, A. Sigov, And A. Sobyanin, Change of defect structure and the resultant anomalies in the properties of substances near phase-transition points, Sov. Phys. - JETP, 49 (1979), pp. 176-188.

[21] A. LunARDI, Analytic semigroups and optimal regularity in parabolic problems, vol. 16 of Progress in Nonlinear Differential Equations and their Applications, Birkhäuser, Basel, 1995.

[22] A. Martinez-Arias and A. Stewart, Molecular principles of animal development, Oxford University Press, New York, 2002. 
[23] C. B. Muratov, P. V. Gordon, and S. Y. Shvartsman, Self-similar dynamics of morphogen gradients, Phys. Rev. E, 84 (2011), pp. 041916 pp. 1-4.

[24] L. Oswald, Isolated positive singularities for a nonlinear heat equation, Houston J. Math., 14 (1988), pp. 543-572.

[25] H. G. Othmer, K. Painter, D. Umulis, and C. Xue, The intersection of theory and application in elucidating pattern formation in developmental biology, Math. Model. Nat. Phenom., 4 (2009), pp. 3-82.

[26] A. C. PONCE, Selected problems on elliptic equations involving measures. Manuscript submitted to the concours annuel 2012 of the Académie royale de Belgique, 2012.

[27] M. H. Protter And H. F. Weinberger, Maximum principles in differential equations, Springer-Verlag, New York, 1984.

[28] P. PuCCI AND J. Serrin, The maximum principle, Birkhäuser, Basel, 2007.

[29] G. T. Reeves, C. B. Muratov, T. Schüpbach, and S. Y. Shvartsman, Quantitative models of developmental pattern formation, Devel. Cell, 11 (2006), pp. 289-300.

[30] E. SAFTENKU, Effects of calretinin on Ca2+ signals in cerebellar granule cells: Implications of cooperative Ca2+ binding, Cerebellum, 11 (2012), pp. 102-120.

[31] A. Sherman, G. Smith, L. Dai, and R. Miura, Asymptotic analysis of buffered calcium diffusion near a point source, SIAM J. Appl. Math., 61 (2001), pp. 1816-1838.

[32] S. ShVARTSMAn AND R. BAKeR, Mathematical models of morphogen gradients and their effects on gene expression, Wiley Interdisciplinary Reviews. Developmental Biology, 1 (2012), pp. $715-730$.

[33] R. A. Smith, Semiconductors, Cambridge University Press, Cambridge, 2nd ed., 1978.

[34] M. E. TAYlor, Partial Differential Equations III: Nonlinear Equations, Springer-Verlag, Berlin, 1996.

[35] F. Tostevin, P. R. ten Wolde, and M. Howard, Fundamental limits to position determination by concentration gradients, PLoS Comput. Biol., 3 (2007), p. e78.

[36] L. VÉron, Singular solutions of some nonlinear elliptic equations, Nonlinear Anal., 5 (1981), pp. 225-242.

[37] O. Wartlick, A. Kicheva, and M. Gonzalez-Gaitan, Morphogen gradient formation, Cold Spring Harbor Perspectives in Biology, 1 (2009), p. a001255.

[38] S. R. Yu, M. Burkhardt, M. Nowak, J. Ries, Z. Petrasek, S. Scholpp, P. Schwille, AND M. BRAND, Fgf8 morphogen gradient forms by a source-sink mechanism with freely diffusing molecules, Nature, 461 (2009), pp. 533-536. 